\title{
FRECUENCIA DE Babesia spp. EN CABALLOS DE MONTERÍA, CÓRDOBA (COLOMBIA)
}

\section{FREQUENCY OF Babesia spp. IN HORSES OF MONTERIA, CORDOBA (COLOMBIA)}

\begin{abstract}
Alfonso Calderón ${ }^{1}$, José Cardona ${ }^{2}$, Óscar Vergara ${ }^{3}$
${ }^{1}$ MVZ, M.Sc. Profesor Titular Universidad de Córdoba, Facultad Medicina Veterinaria y Zootecnia, Instituto de Investigaciones Biológicas del Trópico. Sede Berástegui, km 26 vía Ciénaga de Oro. Montería, Córdoba, Colombia. E-mail: alcaran1@ yahoo.com; ${ }^{2}$ Profesor de Medicina y Clínica de Grandes Animales, Semillero de Estudios e Investigaciones en Medicina de Grandes Animales, Grupo MEGA, Departamento de Ciencias Pecuarias, Universidad de Córdoba, Montería, Colombia. Estudiante de Doctorado en Medicina Veterinaria, Universidad Federal de Viçosa y Bolsista do Programa Estudantes-Convênio de Pós-Graduação - PEC-PG, da CAPES/CNPq, Brasil. E-mail: cardonalvarez@hotmail.com; ${ }^{3}$ Zoot. Dr. Sci. Profesor Asistente Universidad de Córdoba, Facultad Medicina Veterinaria y Zootecnia. Grupo Producción Animal Tropical, e-mail: overgara@ correo.unicordoba.edu.co
\end{abstract}

Rev. U.D.C.A Act. \& Div. Cient. 16(2): 451-458, Julio-Diciembre, 2013

\section{RESUMEN}

La Babesiosis equina o piroplasmosis equina, enfermedad que se transmite a los caballos por las garrapatas, es la principal restricción internacional para la movilización de los equinos. Es producida por los protozoos Theileria equi y Babesia caballi. Además de los caballos, afecta a mulas, asnos y cebras. Por medio de muestreo por conveniencia, se implementó un estudio transversal, donde se seleccionaron seis pesebreras en Montería (Córdoba), en las que se recolectaron 126 muestras de sangre de equinos (Equus ferus caballus), en que se determinó el hematocrito y se colorearon, mediante tinción de Wright, para determinar la frecuencia de Babesiosis. El 18,25\% de las muestras fueron positivas a Babesia spp. Se analizaron diferentes variables, como sistema de alimentación, edad, sexo, color y raza, para ser relacionadas con la frecuencia de Babesia spp. en equinos. La media general del hematocrito (Hto) fue $34,2 \% \pm 6,2$, con un intervalo de confianza (95\%) de 33,1 a $35,2 \%$. Para los equinos negativos, el promedio del Hto fue del $34,5 \% \pm$ 5,2 y, de los positivos, del 32,9 $\pm 9,4 \%$; no se encontraron diferencias significativas entre estos promedios. Se determinó que en los diferentes sistemas de manejo de los equinos en la ciudad de Montería, se presentaron casos de babesiosis equina y se hace necesario implementar un programa integral de control de vectores.

Palabras clave: Equinos, infección, piroplasmosis, tinción de Wright.

\section{SUMMARY}

Equine babesiosis or equine piroplasmosis, disease transmitted to horses by ticks, is the main restriction of international horse mobilization. It is produced by the protozoa Babesia caballi or Theileria equi, which besides horses, affect mules, donkeys and zebras. Through a convenience sample, a cross sectional study was implemented and six stables in Monteria (Cordoba) were selected, taking 126 blood samples of horses (Equus ferus caballus), in which by hematocrit and staining by Wright's stain the frequency of babesiosis was determined. A $18.25 \%$ of the samples were positive for Babesia spp. Different variables such as feeding system, age, sex, color and race were analyzed and related to the frequency of positive Babesia spp. in horses. The general average of hematocrit was $34.2 \pm 6.2 \%$ with a confidence interval (95\%) of 33.1 at $35.2 \%$. For seronegatives horses the average of the hematocrit was $34.5 \pm 5.2 \%$ and for seropositive horses $32.9 \pm 9.4 \%$; no significant difference was found between these averages. In was determined that in the different horse management systems in Monteria cases of equine babesiosis are present and the need of a comprehensive vector control program has to be implemented.

Key words: Equides, infection, piroplasmosis, Wright's stain.

\section{INTRODUCCIÓN}

La Babesiosis equina (BE) o piroplasmosis equina es causada por los protozoos intraeritrocitarios Theileria equi y Babesia caballi y pertenecen al filo Apicomplexa y la orden 
Piroplasmida (APHIS, 2008; Pitel et al. 2010). La Babesia equi fue reclasificada como Th. equi, en 1998 (Mehlhorn \& Schein, 1998). Estos protozoos afectan a caballos, mulas, asnos y cebras (Baldani et al. 2007; APHIS, 2008) y son la principal restricción internacional para la movilización de los equinos (APHIS, 2008).

La transmisión de los protozoos, se produce a través de la picadura (inoculación de esporozoitos) en caballos por un vector (garrapata), que se infectan al ingerir sangre de otros équidos infectados o enfermos de piroplasmosis. Los géneros de las garrapatas: Dermacentor, Hyalomma y Rhipicephalus pueden ser vectores y se desconoce su rol epidemiológico (Hunfeld et al. 2008; APHIS, 2008). Infección por Th. equi, se comprobó en caballos, donde se excluyó diseminación, a partir de la Dermacentor variabilis y se sugirió que el uso compartido de agujas en las transfusiones de sangre puede ser un factor de riesgo de la transmisión (Corto et al. 2012).

Los signos clínicos de BE son variables e inespecíficos. Th. equi produce una forma clínica más grave que la $B$. caballi (CFSPH, 2008), pero incluyen, para ambos protozoos, fiebre, anemia, retardo en el crecimiento, ictericia, emaciación, aumento de las frecuencias cardiacas y respiratorias, cólico, edema en los miembros, hemorragias petequiales en mucosas y, dependiendo de la forma clínica (hiperaguda, aguda o crónica), puede haber signos neurológicos. La lisis intravascular de los eritrocitos produce hemoglobinemia, hiperbilirubinemia, hemoglobinuria (DAFF, 2008; CFSPH, 2008; Rashid et al. 2009).

La Babesiosis es endémica en regiones tropicales y subtropicales (Ikadai et al. 1999; Aguirre et al. 2004; Mantran et al. 2004), como África, Medio Oriente, Asia, América Central y Suramérica, el Caribe (incluso en Puerto Rico), Europa oriental y del sur (Mantran et al. 2004; CFSPH, 2008; APHIS 2008). En áreas templadas no es común su presencia (Mantran et al. 2004), pero se han presentado episodios por el incremento en el transporte de los equinos desde las zonas endémicas (Sigg et al. 2010); por ejemplo, Butler et al. (2012), en Schouwen-Duiveland (isla de la provincia de Zeeland, Holanda), confirmaron transmisión autóctona de $B$. caballi y Th. equi en dos casos agudos y en el $4 \%$ de una muestra de 300 equinos fueron seropositivos para piroplasmosis y concluyen que la presencia de Dermacentor reticulatus, más la importación sin restricciones de zonas endémicas de caballos, fueron las causas de los casos clínicos y de los anticuerpos detectados (Nijhof et al. 2007). Igualmente, Sigg et al. (2010) encontraron una mayor seroprevalencia en caballos importados de Francia, de España y de Portugal, en comparación con caballos nacidos en Suiza.

Esta patología no es endémica en Estados Unidos, porque las especies de garrapatas no portan estos parásitos. Igual situación se presenta en Australia, en Canadá, en Inglaterra, en Islandia, en Irlanda y en Japón (Mantran et al. 2004; CFSPH, 2008; APHIS, 2008). El objetivo fue determinar la frecuencia de Babesia spp. en equinos (E. ferus caballus) de Montería (Córdoba), para implementar más programas de control integral de los vectores.

\section{MATERIALES Y MÉTODOS}

Tipo de estudio: Correspondió a un estudio descriptivo, de corte transversal.

Zona de estudio: Las muestras, se tomaron en Montería, capital del departamento de Córdoba, localizada a los $8^{\circ} 46^{\prime}$ 15" N, 750 51' 31" O, con una altura de 18 m.s.n.m., con una humedad relativa del $85 \%$ y una temperatura promedio de $28^{\circ} \mathrm{C}$.

Selección de la muestra: Por medio de un muestreo por conveniencia, se seleccionaron seis pesebreras, con diferentes sistemas de producción; donde los propietarios y los administradores facilitaron el acceso y los registros de los equinos; la población de estas pesebreras fue 126 ejemplares.

Toma de las muestras: Previa sujeción de los equinos, se tomó una muestra de sangre de $3 \mathrm{ml}$ de la vena transversa facial (Kemble, 1994), en un tubo vacutainer de plástico al vacío, impregnado en su interior de silicona y ácido etilendiaminotetraacético (EDTA) pulverizado. Cada muestra, se rotuló y se conservó en refrigeración a $4^{\circ} \mathrm{C}$, en una nevera de poliestireno.

Las muestras, se procesaron en el Laboratorio Clínico de la Clínica Julio E. Cuervo de la Universidad de Córdoba, sede Berástegui, donde se les determinó el volumen globular agregado (VGA) o hematocrito (Hto) y la tinción de Wright, con el fin de observar el agente etiológico.

La tinción de Wright, se hizo mediante una pequeña gota de sangre que se depositó con una pipeta Pasteur en una lámina portaobjetos y se expandió con un cubreobjetos, con una inclinación de $45^{\circ}$; el extendido se secó y después se aplicó una solución de colorante de Wright por toda la lámina durante 2 minutos; luego, se colocaron 2 o 3 gotas de agua destilada, se sopló hasta que la placa cambió el color tornasol, se dejó en reposo durante 2 minutos, se enjuagó con agua corriente y se procedió a la lectura en un microscopio de luz con el objetivo de 100X, para visualizar el agente etiológico.

La determinación del hematocrito, se hizo mediante un tubo capilar heparinizado (tubo de hematocrito), con aproximadamente un $80 \%$ del volumen del capilar con sangre; se evitó 
la formación de burbujas de aire en el interior del capilar; un extremo se selló con plastilina y el capilar se ubicó en la plataforma de la microcentrífuga, durante 5 minutos, a 10.000rpm. Luego, el capilar se colocó en una tabla de hematocrito graduada, donde el nivel inferior del tubo coincidió con el nivel de la línea horizontal correspondiente a cero; la lectura del hematocrito correspondió a la altura de la separación de la sangre y el plasma que se leyó en un tabla para hematocrito a graduada.

Análisis estadístico: Se elaboró una base de datos en formato Excel, donde se consignó información de las variables de los equinos evaluados y los resultados obtenidos en el laboratorio. Se usó Chi-cuadrado, con el objeto de determinar si las variables sexo, edad, raza, sistema de producción (estabulado, semi estabulado o potrero) eran independientes de la frecuencia de babesia. Los análisis, se realizaron mediante el software SAS.

Aspectos éticos: El Comité de Ética del Instituto de Investigaciones Biológicas del Trópico (IIBT) de la Universidad de Córdoba, clasificó este estudio de bajo riesgo. Las muestras fueron tomadas por estudiantes de la Facultad de Medicina Veterinaria y Zootecnia, supervisados por uno de los autores, quienes tuvieron en cuenta, para los procedimientos de toma de muestra, de manejo y de conservación, las normas éticas, técnicas, científicas y administrativas para la investigación en animales, según lo mencionado en la ley 84 (Congreso Nacional de Colombia, 1989) y lo referido por Mrad (2006). Se mantuvo la confidencialidad de la información de predios muestreados y de animales seropositivos.

\section{RESULTADOS Y DISCUSIÓN}

La población de caballos fue de 126 individuos, donde el 58,73\% (74/126) fueron machos y el 41,27 (52/125) hembras. De acuerdo al sistema de producción, el 69,84\% (88/126), se clasificó como estabulados; el 12,69\% (16/126), semi-estabulados y el $17,47 \%$ (22/126), permanecieron todo el tiempo en potrero.

Mediante la tinción de Wright, la frecuencia de Babesia spp. fue del 18,25\% (23/126). Menores valores absolutos han sido reportados por De Vera et al. (2006), en dos hipódromos en Venezuela (seroprevalencia 13,1\%), mediante inmunofluorescencia indirecta (IFI) y donde este menor valor se atribuyó a los mayores cuidados veterinarios en los hipódromos y por Castellanos et al. (2010), en equinos criollos del estado Apure (prevalencia del Trypanosoma evansi del 7,3\%, para $B$. equi $1,4 \%$ ), usando una técnica muy similar a la del actual estudio y donde no se incluyeron equinos estabulados y semi-estabulados.

Seroprevalencias más altas para Th. equi y B. caballi en comparación con el menor valor absoluto al del presente estudio, han sido reportadas en otros países (Tabla 1). Estas diferencias pueden ser ocasionadas por el uso de técnicas indirectas, que detectan la presencia de anticuerpos, que indican infección activa o infecciones pasadas, a diferencia del presente estudio, donde se implementó una técnica directa que muestra infección activa.

No se encontró efecto significativo $(p>0,05)$ del tipo de producción, respecto a la frecuencia de babesia. Al discriminar la frecuencia por tipo de producción, se encontró que el 10,32\% (13/126) fueron equinos estabulados; el 4,76\% (6/126), semi-estabulados y el 3,17\% (4/126), de potrero. Guillén et al. (2001), en Aragua (Venezuela), no encontraron diferencias significativas entre el tipo de producción, debido a que se limitó la transmisión por el control de vectores. Botteon et al. (2002), en Brasil, hallaron una menor seroprevalencia, posiblemente, al interés en mantener a los equinos seronegativos, con perspectivas de exportación y para la participación en eventos deportivos internacionales. García et al. (2013) determinaron, como factor de riesgo asociado a una mayor seroprevalencia de Th. equi, la presencia de garrapatas y Peckle et al. (2013), en Rio de Janeiro (Brasil), determinaron que la presencia de $A$. cajennense fue un factor de riesgo $(\mathrm{OR}=4,1 ; \mathrm{IC}=1,8$ a 9,1$)$, la seroprevalencia para $T$. equi fue del $81 \%$ y concluyeron que $A$. cajennense es un vector biológico para Th. equi.

Respecto al sexo, la frecuencia de babesia fue independiente ( $p>0.05)$ de éste. La presencia de Babesia en equinos fue mayor en machos (14,28\%; $n=18 / 126)$ y menor en las hembras (3,97\%; $n=5 / 126)$. Hornok et al. (2007), en Hungría, Golynski et al. (2008), en Brasil, Karatepe et al. (2009), en Turquía, Sigg et al. (2010), en Suiza, Grandi et al. (2011), en Italia y Farkas et al. (2013), en Hungría, no encontraron diferencias estadística entre seropositividad y sexo.

La presencia de babesia fue independiente de la edad $(p>0.05)$ de los animales evaluados. En todas las edades de los equinos, se determinaron anticuerpos (Tabla 2), pero la mayor frecuencia se observó en el grupo de 3 a 6 años. Hornok et al. (2007), en Hungría, Golynski et al. (2008), en Brasil, Sigg et al. (2010), en Suiza, Grandi et al. (2011) en Italia y Farkas et al. (2013), en Hungría, no determinaron diferencias estadística entre seropositividad y la edad. García et al. (2013) establecieron que el incremento en la edad también fue un factor de riesgo asociado con una mayor seroprevalencia de Th. equi.

El color y la raza (Tabla 3) de los equinos evaluados también se comportaron como variables independientes, respecto a la frecuencia de babesiosis ( $p>0.05)$. Golynski et al. (2008), en Brasil, no encontraron diferencias estadísticamente significativas entre razas. 
Tabla 1. Sero/Prevalencia de Babesia caballi y Theileria equi en equinos de diferentes países, durante los últimos cinco años.

\begin{tabular}{|c|c|c|c|c|}
\hline País & Región & Sero/Prevalencia & Técnica & Autor \\
\hline Turquía & Mar Negro & $\begin{array}{l}\text { B. caballi } 34,6 \% \\
\text { Th. equi } 21,59 \% \\
\text { I. mixta } 5,2 \%\end{array}$ & IFI & Acici et al. 2008 \\
\hline Hungría & & B. caballi $6,19 \%$ & Elisa-C & Hornok et al. 2007 \\
\hline Brasil & R. Grande do Sul & $\begin{array}{l}\text { B. equi } 31,6 \% \\
\text { B. equi } 35,8 \%\end{array}$ & $\begin{array}{l}\text { Elisa } \\
\text { IFA }\end{array}$ & Golynski et al. 2008 \\
\hline Suráfrica & & $\begin{array}{l}\text { Th. equi } 96,5 \% \\
\text { Th. equi } 95,2 \% \\
\text { Th. equi } 100\end{array}$ & & Motloang et al. 2008 \\
\hline Turquía & & $\begin{array}{l}\text { Th. equi } 12,8 \% \\
\text { B. caballi } 9,6 \%\end{array}$ & IFI & Karatepe et al. 2009 \\
\hline Brasil & São Paulo & $\begin{array}{l}\text { Th. equi } 54,1 \% \\
\text { B. caballi } 21,6\end{array}$ & Elisa-C & Kerber et al. 2009 \\
\hline Suiza & & $\mathrm{EPP}=7,3 \%$ & IFI & Sigg et al. 2010 \\
\hline Italia & Norte del país & $\begin{array}{l}\text { Th. equi } 8,2 \% \\
\text { B. caballi } 0,3 \%\end{array}$ & IFI & Grandi et al. 2011 \\
\hline Mongolia & & Th. equi $67 \%$ & Frotis & Sloboda et al .2011 \\
\hline Corea & & Th. equi $1,1 \%$ & Elisa-C & Seo et al. 2011 \\
\hline Jordania & & Th. equi $14,1 \%$ & Elisa-C & Abutarbush et al. 2012 \\
\hline México & Nor-este & $\begin{array}{l}\text { Th. equi } 45,2 \% \\
\text { B. caballi } 27,4 \% \text {, }\end{array}$ & IFI & Cantú et al. 2007 \\
\hline España & Andalucía & $\begin{array}{l}\text { B. caballi, } 11,4 \% \\
\text { Th. equi } 50,3 \% \\
\text { I. mixta } 8,4 \%\end{array}$ & Elisa-C & García et al. 2013 \\
\hline Mongolia & & $\begin{array}{l}\text { B. caballi, } 51,6 \% \\
\text { Th. equi } 19,6 \% \text {, } \\
\text { I. mixtas } 10,4 \% \text {. }\end{array}$ & Elisa & Munkhjargal et al. 2013 \\
\hline
\end{tabular}

I. mixta $=B$. caballi + Th. equi

Tabla 2: Frecuencia de Babesia spp. en equinos de Montería, según la edad.

\begin{tabular}{|c|c|c|c|c|c|}
\hline \multirow{2}{*}{ Edad/años } & \multirow{2}{*}{$\mathbf{n}$} & \multicolumn{2}{|c|}{ Positivos } & \multicolumn{2}{c|}{ Negativos } \\
\cline { 3 - 6 } & & $\mathbf{n}$ & $\mathbf{\%}$ & $\mathbf{N}$ & $\mathbf{\%}$ \\
\hline $0-3$ & 38 & 5 & 3,96 & 33 & 26,19 \\
\hline $3-6$ & 44 & 9 & 7,15 & 35 & 27,77 \\
\hline $6-9$ & 32 & 6 & 4,76 & 26 & 20,63 \\
\hline$>9$ & 12 & 3 & 2,38 & 9 & 7,15 \\
\hline Total & $\mathbf{1 2 6}$ & $\mathbf{2 3}$ & $\mathbf{1 8 , 2 5}$ & $\mathbf{1 0 3}$ & $\mathbf{8 1 , 7 4}$ \\
\hline
\end{tabular}


Tabla 3: Frecuencia de Babesia spp. en equinos de Montería, según raza.

\begin{tabular}{|l|c|c|c|c|c|}
\hline \multirow{2}{*}{\multicolumn{1}{c|}{ Raza }} & & \multicolumn{2}{c|}{ Positivos } & \multicolumn{2}{c|}{ Negativos } \\
\cline { 2 - 6 } & $\mathbf{n}$ & $\mathbf{n}$ & $\mathbf{\%}$ & $\mathbf{n}$ & $\mathbf{\%}$ \\
\hline Criollo & 92 & 13 & 10,31 & 79 & 62,69 \\
\hline Media sangre inglés & 1 & 1 & 0,79 & 0 & 0 \\
\hline Mestizo argentino & 19 & 7 & 5,55 & 12 & 9,52 \\
\hline Pura sangre inglés & 1 & 0 & 0 & 1 & 0,79 \\
\hline Pony & 9 & 1 & 0,79 & 8 & 6,41 \\
\hline Silla Argentina & 4 & 1 & 0,79 & 3 & 2,38 \\
\hline Total & $\mathbf{1 2 6}$ & $\mathbf{2 3}$ & $\mathbf{1 8 , 2 3}$ & $\mathbf{1 0 3}$ & $\mathbf{8 1 , 7 9}$ \\
\hline
\end{tabular}

La media general del volumen globular agregado (VGA) o hematocrito (Hto) fue de $34,2 \pm 6,2 \%$, con un intervalo de confianza (95\%), entre 33,1 a 35,2\%. En los equinos negativos, el promedio del \% Hto fue del 34,5 $\pm 5,2 \%$ y de los positivos del $32,9 \pm 9,4 \%$. No se encontró diferencias significativas entre el valor del Hto de equinos positivos y negativos. El valor promedio del \% Hto en equinos sanos criollos, reportados en el valle de Urabá, fluctuó 32 y $47 \%$ (Castillo et al. 2010); sin embargo, se desconoce un valor de referencia para equinos criollos sanos, en las condiciones del medio Sinú.

Resultados contradictorios, en cuanto al valor del Hto, han sido reportados por Aguirre et al. (2004), en Salta (Argentina), quienes determinaron un mayor valor (Hto del $40 \%$ ), en equinos afectados por babesiosis; aunque no aclaran la causa de este valor, puede ser consecuencia de la deshidratación y la concentración del paquete celular.

Valores muy similares del Hto reportado en el presente estudio ha sido reportado por Castillo et al. (2006), en Cuba, en equinos destinados a la tracción, donde el promedio del Hto fue de 32,52 $\pm 3,0 \%$ y donde evidenciaron un estado anémico por el intenso trabajo. Valores inferiores al del presente estudio fue reportado por Castellanos et al. (2010), con un valor del $31,17 \pm 9,91 \%$, y tan sólo el 1,4\% estaban anémicos e infectados por $B$. equi.

Rashid et al. (2009), en Pakistán, hallaron una disminución del hematocrito. Hailat et al. (1997), Butler et al. (2005), Camacho et al. (2005) afirmaron que dentro de los hemoparásitos $B$. equi puede ser una causa de anemia severa, a diferencia de Sigg et al. (2010), quienes no encontraron asociaciones entre la pérdida de peso y la seroprevalencia, por Th. equi y $B$. caballi. Se encontró que en los sistema de manejo (estabulados, semi-estabulados y en pastoreo) de los equinos, en Montería, se encuentran casos de babesiosis equina y se hace necesario implementar un programa integral de control de vectores.
Agradecimientos. Los autores agradecen a los propietarios y a los administradores de las pesebreras, donde se adelantó el estudio, así como también a los estudiantes Juan Diego Torralvo y Juan Pablo Martínez, sin cuya colaboración no sería posible haber adelantado el estudio. Conflicto de intereses: El manuscrito fue preparado y revisado con la participación de todos los autores, quienes declaramos que no existe ningún conflicto de intereses, que ponga en riesgo la validez de los resultados presentados.

\section{BIBLIOGRAFÍA}

1. ABUTARBUSH, S.M.; ALQAWASMEH, D.M.; MUKBEL, R.M.; AL MAJALI, A.M. 2012. Equine babesiosis: seroprevalence, risk factors and comparison of different diagnostic methods in Jordan. Transbound Emerg. Dis. 59(1):72-78.

2. ACICI, M.; UMUR, S.; GUIEVENC, T.; ARSLAN, H.H.; KURT, M. 2008. Seroprevalence of equine babesiosis in the Black Sea region of Turkey. Int. Parasitol. 57(2):198-200.

3. AGUIRRE, D.; CARFUNE, M.; RADA, M.; TORINIO De, E.S. 2004. Babesiosis clínica en equinos de Cerrillos, Salta, Argentina. Rev. RIA. 33(003):123-133.

4. ANIMAL AND PLANT HEALTH INSPECTION SERVICE (APHIS). 2008. Piroplasmosis equina. Disponible desde Internet en:

http://www.aphis.usda.gov/publications/animal health/content/printable_version/fs_equine_piro_ sp.pdf (con acceso 20/02/2013).

5. BALDANI, D.; MACHADO, R.; RASO, T.; PINTO, A. 2007. Serodiagnosis of Babesia equi in horses submitted to exercise stress. Pesq. Vet. Bras. 27(4):179-183. 
6. BOTTEON, P de T.L.; MASSARD, C.L.; BOTTEON, R de C.M.; LOSS, Z.G.; LINHARES, G.F.C. 2002. Seroprevalencia de Babesia equi en tres diferentes sistemas de criança de equinos. Rio de Janeiro - Brasil. Parasitol. Latinoam. 57:141-145.

7. BUTLER, C.; Van GILS, J.; Van Der KOLK, J. 2005. A literature review of equine piroplasmosis after an episode of acute babesiosis in dutch standardbred foal after a stay in Normandy. Tijdschr. Diergeneeskd. 130(23):726-731.

8. BUTLER, C.M.; SLOET Van OLDRUITENBORGH, O.M.M.; STOUT, T.A.; Van Der KOLK, J.H.; WOLLENBERG, L.V.; NIELEN, M.; JONGEJAN, F.; WERNERS, A.H.; HOUWERS, D.J. 2012. Prevalence of the causative agents of equine piroplasmosis in the South West of The Netherlands and the identification of two autochthonous clinical Theileria equi infections. Vet. J. 193(2):381-385.

9. CAMACHO, A.; GUITIAN, F.; PALLAS, E., GESTAL, J.; OLMEDA, A.; HABELA, M.; TELFORD, S.; SPIELMAN, A. 2005. Theileria (Babesia) equi and Babesia caballi infections in horses in Galicia, Spain. Trop. Anim. Health Prod. 37(4):293-302.

10. CANTÚ, A.; ORTEGA, S.J.A.; MOSQUEDA, J.; GARCIA, V.Z., HENKE, S.E.; GEORGE, J.E. 2007. Immunologic and molecular identification of Babesia bovis and Babesia bigemina in free ranging white-tailed deer in northern Mexico. J. Wildl. Dis. 43(3):504507.

11. CASTELLANOS, R.; CANELÓN, J.L.; CALZOLAIO, V.; AGUINACO, F.; LÓPEZ, Á.; MONTESINOS, R. 2010. Estudo hematológico y detección de hemoparásitos en caballos criollos venezolanos de dos hatos del Estado Apure, Venezuela. Rev. Cient. 20(2):153-160.

12. CASTILLO, F.C.A.; TOBÓN, R.M.; CANO, B.C.A.; MIRA, H.J.; SUÁREZ, O.A.P.; VÁSQUEZ, E.M. 2010. Valores hematológicos en caballos criollos colombianos del Valle de Aburrá. Disponible desde Internet en: http://repository.lasallista.edu.co/dspace/ bitstream/10567/73/1/245-262.pdf (con acceso 20/02/2013).

13. CASTILLO, J.; CEPERO, O.; SILVEIRA, E.; CASANOVA, R.; QUIÑONES, R.; MONTEAGUDO, E.; GUTIÉRREZ, I. 2006. Caballos de tracción de la ciudad de Santa Clara, Cuba. II Algunos parámetros hematológicos. Rev. Electr. Vet. REDVET VII (9):1-5. Disponible desde Internet en: http://www.veterinaria.org/ revistas/redvet/n090906/ 090617.pdf (con acceso 20/02/2013)

14. CENTER FOR FOOD SECURITY AND PUBLIC HEALTH (CFSPH). 2008. Equine piroplasmosis. Iowa State University and Institute for International Cooperation in Animal Biologics. Disponible desde Internet en: http://www.cfsph.iastate.edu/Factsheets/es/piroplasmosis_equina.pdf. (con acceso 20/02/2013).

15. CONGRESO NACIONAL DE COLOMBIA. 1989. Ley 84. Por la cual se adopta el estatuto nacional de protección de los animales y se crean unas contravenciones y se regula ll referente a su procedimiento y competencia. República de Colombia, gobierno nacional. Diario Oficial 39120 de diciembre 27 de 1989. Disponible desde Internet en: http://www.alcaldiabogota.gov.co/sisjur/normas/Norma1.jsp?i=8242 (con acceso 20/02/2013).

16. CORTO, M.A.; CLARK, C.K.; HARVEY, J.W.; WENZLOW, N.; HAWKINSS, I.K.; ALLRED, D.R.; KNOWLES, D.P.; CORN, J.L.; GRAUSE, J.F.; HENNAGER, S.G.; KITCHEM, D.L.; TRAUB, D.; JOSUE, L. 2012. Outbreak of equine piroplasmosis in Florida. J. Am. Vet. Med. Assoc. 240(5):588-595.

17. De VERA, M.; GUILLÉN, A.T.; GARCÍA, F.; CONTRERAS, R.; SIERRALTA, A.; LEÓN, E. 2006. Seroprevalencia de la babesiosis equina en caballos pura sangre de carrera alojados en los hipódromos La Rinconada y Nacional de Valencia, Venezuela. Vet. Trop. 31(12):43-52.

18. DEPARTMENT AGRICULTURE FISHERIES AND FORESTRY (DAFF). 2008. Exotic animal diseases bulletin. Equine piroplasmosis. Austral. Vet. J. 86(11):2021.

19. FARKAS, R.; TÁNCZOS, B.; GYURKOVSZKY, M.; FÖLDVÁRI, G.; SOLYMOSI, N.; EDELHOFER, R.; HORNOK, S. 2013. Serological and molecular detection of Theileria equi infection in horses in Hungary. Vet Parasitol. 192(1-3):143-138

20. GARCÍA, B.I.; ARENAS, M.A.; HERNÁNDEZ, E.; ADASZEK, L.; CARBONERO, A.; ALMERIA, S.; TÉLLEZ, J.J.A.; PALOMINO, G.P.; ARENAS, A. 2013. Seroprevalence and risk factors associated with Babesia caballi and Theileria equi infection in equids. Vet $\mathrm{J}$. 195(2):172-178.

21. GOLYNSKI, A.A.; FERNANDES, K.R.; BALDANI, C.D.; GOLYNSKI, A.L.; MACHADO, R.Z.; BOTTEON, P. 
DE T., MASSARD, C.L. 2008. Seroepidemiological studies on Babesia equi in horses from the state of Rio Grande do Sul determined by indirect immunoflourecence test and Elisa. Parasitol. Vet. 186(34):461-465.

22. GRANDI, G.; MOLINARI, G.; TITTARELLI, M.; SASSERA, D.; KRAMER, L.H. 2011. Prevalence of Theileria equi and Babesia caballi infection in horses from northern Italy. Vector Borne Zoonotic Dis. 11(7):955956.

23. GUILLÉN, A.T.T.; LEÓN, A.E.A.; ARAGORT, F.W.; SILVA, M. 2001. Diagnóstico de hemoparásitos en el Instituto de Investigaciones Veterinarias. Período 19862000. Vet. Trop. 26(1):47-62.

24. HAILAT, N.Q.; LAFI, S.Q.; DARRAJI, A.M.; Al ANI, F.K. 1997. Equine babesiosis associated with strenuous exercise: Clinical and pathological studies in Jordan. Vet. Parasitol. 69(1-2):1-8.

25. HORNOK, S.; EDELHOFER, R.; FÖLDVÁRI, G.; JOACHIM, A.; FARKAS R. 2007. Serological evidence for Babesia canis infection of horses and an endemic focus of $B$. caballi in Hungary. Acta Vet. Hung. 55(4):491-500.

26. HUNFELD, K.P.; HILDEBRANDT, A.; GRAY, J.S. 2008. Babesiosis: Recent insights into an ancient disease. Int. J. Parasitol. 38:1219-1237.

27. IKADAI, H.; TAMAKI, X.; XUAN, I.; IGARASHI, I.; KAWAI, S.; NAGASAWA, H.; FUJISAKI, K., TOYODA, Y.; SUZUKI, N.; MIKAMI, T. 1999. Inhibitory effect of monoclonal antibodies on the growth of Babesia caballi. Int. J. Parasitol. 29:1785-1791.

28. KARATEPE, B.; KARATEPE, M.; CAKMAK, A.; KARAER, Z.; ERGÜN, G. 2009. Investigation of seroprevalence of Theileria equi and Babesia caballi in horses in Nigde province, Turkey. Trop Anim Health Prod. 41(1):109-113.

29. KEMBLE, T.J. 1994. Alternative site to the jugular vein for serial blood sampling. Equine Vet. J. 6(6):301-302.

30. KERBER, C.E.; LABRUNA, M.B.; FERREIRA, F.; DE WAAL, D.T.; KNOWLES, D.P.; GENNARI, S.M. 2009. Prevalence of equine piroplasmosis and its association with tick infestation in the State of São Paulo, Brazil. Rev. Bras. Parasitol. Vet. 18(4):1-8.
31. MANTRAN, A.; VOTION, D.M.; AMORY, H. 2004. Piroplasmosis: A problem in Belgium? In: Proc. Annual Congress Belgian Equine Practitioners Society, Liege, Belgium. Disponible desde Internet en: http:// www.ivis.org/proceedings/BEPS/2004/Amory_nl/ivis. pdf. con acceso 20/02/2013).

32. MEHLHORN, H.; SCHEIN, E. 1998. Redescription of Babesia equi Laveran, 1901 as Theileria equi Mehlhorn, Schein. Parasitol. Res. 84(6):467-475.

33. MOTLOANG, M.Y.; THEKISOE, O.M.; ALHASSAN, A.; BAKHEIT, M.; MOTHEO, M.P.; MASANGANE, F.E.; THIBEDI, M.L.; INOUE, N.; IGARASHI, I.; SUGIMOTO, C.; MBATI, P.A. 2008. Prevalence of Theileria equi and Babesia caballi infections in horses belonging to resource-poor farmers in the north-eastern Free State Province, South Africa. Onderstepoort. J. Vet. Res. 75(2):141-146.

34. MRAD, A. 2006. Ética en la investigación con modelos animales experimentales. Alternativas y las 3 RS de Russel. Una responsabilidad y un compromiso ético que nos compete a todos. Rev. Col. Bioét. 1(1):163184.

35. MUNKHJARGAL, T.; SIVAKUMAR, T.; BATTSETSEG, B.; NYAMJARGAL, T.; ABOULAILA, M.; PUREVTSEREN, B.; BAYARSAIKHAN, D.; BYAMBAA, B.; TERKAWI, M.A.; YOKOYAMA, N.; IGARASHI, Y.O. 2013. Prevalence and genetic diversity of equine piroplasms in Tov province, Mongolia. Infect. Genet. Evol. 16C:178-185.

36. NIJHOF, A.M.; BODAAN, C.; POSTIGO, M.; NIEUWENHUIJS, H.; OPSTEEGH, M.; FRANSSEN, L.; JEBBINK, F.; JONGEJAN, F. 2007. Ticks and associated pathogens collected from domestic animals in the Netherlands. Vector-Borne Zoon. Dis. 7:585-595.

37. PECKLE, M.; PIRES, S.M.; Dos SANTOS, M.T.; ROIER, C.R.E.; Da SILVA, B.C.; VILELA, A.R.J.; SANTOS, A.H.; MASSARD, L.C.; 2013. Molecular epidemiology of Theileria equi in horses and their association with possible tick vectors in the state of Rio de Janeiro, Brazil. Parasitol. Res. 112:2017-2025.

38. PITEL, P.H.; PRONOST, S.; SCRIVE, T.; LÉON, A.; RICHARD, E.; FORTIER, G. 2010. Molecular detection of Theileria equi and Babesia caballi in the bone marrow of asymptomatic horses. Vet Parasitol. 170(1-2):182-184. 
39. RASHID, A.; MUBARAK, A.; HUSSAIN, A. 2009. Babesiosis in equines in Pakistan: a clinical report. Vet. Italiana. 45(3):391-95.

40. SEO, M.G.; YUN, S.H.; CHOI, S.K.; CHO, G.J., PARK, Y.S.; KWON, O.D.; CHO, K.H.; KIM, T.H.; JEONG, K.S.; PARK, S.J.; KWON, Y.S.; KWAK, D. 2011. Seroprevalence of equine piroplasms in the Republic of Korea. Vet. Parasitol. 179(1-3):224-226.

41. SIGG, L.; GERBER, V.; GOTTSTEIN, B.; DOHERR, M.G.; FREY, C.F. 2010. Seroprevalence of Babesia caballi and Theileria equi in the Swiss horse population. Parasitol. Int. 59(3):313-317.
42. SLOBODA, M.; JIRKU, M.; LUKEŠOVÁ, D.; QABLAN, M.; BATSUKH, Z.; FIALA, I.; HOŘÍN, P.; MODRÝ, D.; LUKEŠ, J. 2011. A survey for piroplasmids in horses and bactrian camels in North-Eastern Mongolia. Vet. Parasitol. 179(1-3):246-249.

43. STATISTICAL ANALYSIS SUSTEMS (SAS). 2001. User's guide (versión 9.1) Cary (USA). Institute SAS/STAT.

Recibido: Abril 12 de 2013

Aceptado: Agosto 26 de 2013

Como citar:

Calderón, A.; Cardona, J.; Vergara, Ó. 2013. Frecuencia de babesia spp. En caballos de Montería, Córdoba (Colombia). Rev. U.D.C.A Act. \& Div. Cient. 16(2): 451-458. 